\title{
Circumstellar dust shells of hot post-AGB stars ${ }^{\star}$
}

\author{
G. Gauba and M. Parthasarathy
}

\begin{abstract}
Indian Institute of Astrophysics, Koramangala, Bangalore 560034, India
\end{abstract}
Received 24 June 2003 / Accepted 3 December 2003

\begin{abstract}
Parameters of the circumstellar dust shells of 15 hot post-AGB stars have been derived using a radiative transfer code (DUSTY). Combining the optical, near and far-infrared (ISO, IRAS) data of the stars, we have reconstructed their spectral energy distributions (SEDs) and estimated the dust temperatures, mass loss rates, angular radii of the inner boundary of the dust envelopes and the distances to these stars. The mass loss rates $\left(10^{-6}-10^{-5} M_{\odot} \mathrm{yr}^{-1}\right)$ are intermediate between stars at the tip of the AGB and the PN phase. We have also studied the ISO spectra of 7 of these stars. Amorphous and crystalline silicate features were observed in IRAS 14331-6435 (Hen3-1013), IRAS 18062+2410 (SAO 85766) and IRAS 22023+5249 (LSIII +5224) indicating oxygen-rich circumstellar dust shells. The presence of unidentified infrared (UIR) band at $7.7 \mu, \mathrm{SiC}$ emission at $11.5 \mu$ and the " $26 \mu$ " and "main $30 \mu$ " features in the ISO spectrum of IRAS 17311-4924 (Hen3-1428) suggest that the central star may be carbon-rich. The ISO spectrum of IRAS 17423-1755 (Hen3-1475) shows a broad absorption feature at $3.1 \mu$ due to $\mathrm{C}_{2} \mathrm{H}_{2}$ and/or $\mathrm{HCN}$ which is usually detected in the circumstellar shells of carbon-rich stars.
\end{abstract}

Key words. stars: AGB and post-AGB - stars: early-type - stars: evolution - stars: circumstellar matter - infrared: stars

\section{Introduction}

In the evolution of low and intermediate mass stars $\left(0.8-8 M_{\odot}\right)$, the post-asymptotic giant branch (post-AGB) or protoplanetary nebula (PPN) phase is a transition stage from the tip of the AGB to the planetary nebula (PN) stage (Kwok 1993). The hot postAGB stars form an evolutionary link between the cooler G, F, A supergiant post-AGB stars (Parthasarathy \& Pottasch 1986) and the hotter O-B central stars of PNe (Parthasarathy 1993a).

Analysis of the UV(IUE) spectra of hot post-AGB stars (Gauba \& Parthasarathy 2003), revealed that in many cases, the hot $(\mathrm{OB})$ central stars of PPNe are partially obscured by circumstellar dust shells. Stars on the AGB and beyond are characterised by severe mass loss $\left(10^{-8}-10^{-3} M_{\odot} \mathrm{yr}^{-1}\right)$ which results in the formation of circumstellar envelopes. The physical mechanisms responsible for the intensive mass loss from AGB stars are not well understood although the most promising mechanism to date involves radiation pressure on the dust grains (Tielens 1983). While AGB stars appear to have spherically symmetric dust outflows (e.g. Habing \& Blommaert 1993), PN tend to have axially symmetric inner regions and spherical outer halos (e.g. Schwarz et al. 1992). Inorder to understand the mass loss mechanisms, wind velocities and time scales responsible for the evolution of $\mathrm{PNe}$, we need to study

Send offprint requests to: $\mathrm{G}$. Gauba,

e-mail: gsarkar@iucaa.ernet.in

* Based on observations with ISO, an ESA project with instruments funded by ESA Member States (especially the PI countries: France, Germany, The Netherlands and the UK) and with the participation of ISAS and NASA. the circumstellar environment of the stage intermediate between the AGB and the PN phase, i.e. the post-AGB/PPN phase. Circumstellar dust shells of some cooler post-AGB stars (e.g. Hoogzaad et al. 2002; Hony et al. 2003) and PNe (e.g. Siebenmorgen et al. 1994) have been modelled to derive the dust composition, mass loss rates and dynamical ages.

As a consequence of dredge-up of byproducts of helium burning to the surface of stars on the AGB, the oxygen-rich atmospheres of some of these stars may be transformed into carbon-rich atmospheres (see e.g. Iben \& Renzini 1983). This change of chemistry would also be reflected in the composition of the dust grains formed in the cirumstellar envelopes of AGB and post-AGB stars. With the resolution and wavelength coverage of the ISO mission (Kessler et al. 1996) the detection of prominent gas and solid state features specific to oxygen-rich and carbon-rich chemistries became possible. Amorphous and crystalline silicate features and crystalline water have been reported in the ISO spectra of some AGB and post-AGB stars and the nebulae surrounding [WC] central stars of PNe (see e.g. Waters \& Molster 1999; Hoogzaad et al. 2002). Hrivnak et al. (2000) detected the " $21 \mu$ " and " $30 \mu$ " emission features besides the unidentified infrared (UIR) emission bands at 3.3, $6.2,7.7$ and $11.3 \mu$ in the ISO spectra of a sample of carbon-rich PPNe.

Bogdanov (2000, 2002, 2003) modelled the complete spectral energy distribution (SED) of three hot post-AGB stars, IRAS 18062+2410 (SAO 85766), IRAS 19590-1249 (LSIV-12 111) and IRAS 20462+3416 (LSII+34 26) using radiative transfer codes and derived their mass loss rates, inner radii of the dust envelopes, optical depth of the envelopes and 
Table 1. Hot post-AGB stars.

\begin{tabular}{cccccccccc}
\hline \hline Star No. & IRAS & Name & Optical & $l$ & $b$ & \multicolumn{3}{c}{ IRAS fluxes (Jy.) } \\
\cline { 7 - 10 } & & & Sp. type & & & $12 \mu$ & $25 \mu$ & $60 \mu$ & $100 \mu$ \\
\hline 1. & $12584-4837$ & Hen3-847 & Be $^{1}$ & 304.60 & +13.95 & 36.07 & 48.75 & 13.04 & 3.31 \\
2. & $13266-5551$ & -555588 & B1Ibe & 308.30 & +6.36 & 0.76 & 35.90 & 35.43 & 11.66 \\
3. & $14331-6435$ & Hen3-1013 & B3Ie & 313.89 & -4.20 & 4.04 & 108.70 & 70.71 & 20.61 \\
4. & $16206-5956$ & SAO 243756 & AOIa $^{2}$ & 326.77 & -7.49 & $0.36 \mathrm{~L}$ & 11.04 & 12.30 & 4.83 \\
5. & $17203-1534$ & & B1IIIpe & 8.55 & +11.49 & 0.32 & 10.70 & 6.88 & 3.37 \\
6. & $17311-4924$ & Hen3-1428 & B1IIe & 341.41 & -9.04 & 18.34 & 150.70 & 58.74 & 17.78 \\
7. & $17423-1755$ & Hen3-1475 & Be & 9.36 & +5.78 & 7.05 & 28.31 & 63.68 & 33.43 \\
8. & $17460-3114$ & SAO 209306 & O8III & 358.42 & -1.88 & 6.26 & 20.82 & 12.20 & $220.40 \mathrm{~L}$ \\
9. & $18023-3409$ & LSS 4634 & B2IIIe & 357.61 & -6.31 & $0.26 \mathrm{~L}$ & 2.94 & 1.82 & $25.64 \mathrm{~L}$ \\
10. & $18062+2410$ & SAO 85766 & B1I & 50.67 & +19.79 & 3.98 & 19.62 & 2.90 & $1.00 \mathrm{~L}$ \\
11. & $18371-3159$ & LSE 63 & B1Iabe & 2.92 & -11.82 & $0.25 \mathrm{~L}$ & 6.31 & 5.16 & 1.95 \\
12. & $18379-1707$ & LSS 5112 & B1IIIpe & 16.50 & -5.42 & 1.67 & 23.76 & 7.12 & $3.66 \mathrm{~L}$ \\
13. & $19590-1249$ & LSIV-12 111 & B1Ibe & 29.18 & -21.26 & $0.29:$ & 10.26 & 6.45 & $1.77:$ \\
14. & $22023+5249$ & LSIII +5224 & B $^{4}$ & 99.30 & -1.96 & 1.02 & 24.69 & 14.52 & $3.93 \mathrm{~L}$ \\
15. & $22495+5134$ & LSIII +5142 & PN $^{5}$ & 104.84 & -6.77 & 0.54 & 12.37 & 7.18 & 3.12 \\
\hline
\end{tabular}

A colon : indicates moderate quality IRAS flux, $\mathrm{L}$ is for an upper limit.

The spectral types are from Parthasarathy et al. (2000a) except ${ }^{1}$ Kazarovets et al. (2000); ${ }^{2}$ Schild et al. (1983); ${ }^{3}$ Parthasarathy et al. (2000b); ${ }^{4}$ Simbad database; ${ }^{5}$ Acker et al. (1992).

Table 2. Log of ISO observations.

\begin{tabular}{ccccccc}
\hline \hline IRAS & Name & Date of Obs. & Duration of Obs.(s) & TDT $^{a}$ & Mode $^{b}$ & Speed $^{c}$ \\
\hline $14331-6435$ & Hen3-1013 & 14 July 1997 & 3454 & 60600607 & SWS01 & 3 \\
$16206-5956$ & SAO 243756 & 6 Sep. 1996 & 6538 & 29401311 & SWS01 & 4 \\
$17311-4924$ & Hen3-1428 & 28 Feb. 1996 & 1834 & 10300636 & SWS01 & 2 \\
$17423-1755$ & Hen3-1475 & 17 March 1997 & 1140 & 48700267 & SWS01 & 1 \\
& & 17 March 1997 & 800 & 48700168 & LWS01 & - \\
$18062+2410$ & SAO 85766 & 18 Feb. 1997 & 1140 & 46000275 & SWS01 & 1 \\
$22023+5249$ & LSIII +5224 & 5 Jan. 1997 & 1140 & 41600993 & SWS01 & 1 \\
$22495+5134$ & LSIII +5142 & 5 Jan. 1997 & 1140 & 41601295 & SWS01 & 1 \\
\hline
\end{tabular}

${ }^{a}$ TDT number uniquely identifies each ISO observation. ${ }^{b}$ SWS observing mode used (see de Graauw et al. 1996). ${ }^{c}$ Speed corresponds to the scan speed of observation.

the distances to these stars. We need to study a bigger sample of such stars to understand the evolution of the infrared spectrum as the stars evolve from the cooler post-AGB phase to the hot central stars of PNe. In this paper, we have used the radiative transfer code, DUSTY (Ivezić et al. 1999) to model the circumstellar dust shells of 15 hot post-AGB stars. Additionally, 7 stars from our list were found to have ISO spectra. We also report the analysis of the ISO spectra of these stars.

\section{Target selection}

The hot post-AGB stars for this study (Table 1) were selected from the papers of Parthasarathy \& Pottasch (1989), Parthasarathy (1993b) and Parthasarathy et al. (2000a). High and low resolution optical spectra have confirmed the postAGB nature of several of these stars, e.g. IRAS 17119-5926 (Hen3-1357; Parthasarathy et al. 1993c, 1995; Bobrowsky et al. 1998), IRAS 18062+2410 (SAO 85766; Parthasarathy et al. 2000b; Mooney et al. 2002; Ryans et al. 2003), IRAS 19590-1249 (LSIV-12 111; McCausland et al. 1992;
Conlon et al. 1993a, 1993b; Mooney et al. 2002; Ryans et al. 2003) and IRAS 20462+3416 (LSII+34 26; Parthasarathy 1993a; García-Lario et al. 1997a). The UV(IUE) spectra of some hot post-AGB stars listed in Table 1 showed violet shifted P-Cygni profiles of CIV and NV indicating stellar wind and post-AGB mass loss (Gauba \& Parthasarathy 2003).

\section{ISO observations}

The ISO data archive was searched for spectra of the hot postAGB stars listed in Table 1. Six of the fifteen sources were found to have only SWS (Short Wavelength Spectrometer) spectra while one source, IRAS 17423-1755 (Hen3-1475) had both SWS and LWS (Long Wavelength Spectrometer) spectra. The log of the observations is given in Table 2. ISO SWS spectra have a wavelength coverage from $2.38-45.2 \mu$. The LWS spectra extend from 43-197 $\mu$.

Our objects were observed in the low resolution (AOT 01) mode of the SWS instrument (de Graauw et al. 1996). A spectrum scanned with SWS contains 12 subspectra, that each 
consist of two scans, one in the direction of decreasing wavelength ("up" scan) and the other in the direction of increasing wavelength ("down" scan). There are small regions of overlap in the wavelength between the sub-spectra. Each sub-spectrum is recorded by 12 independent detectors.

The LWS observations were carried out in LWS01 mode, covering the full spectral range at a resolution $(\lambda / \Delta \lambda)$ of $\sim 200$. The characteristics of the ISO LWS instrument are described in Clegg et al. (1996) and the calibration of the instrument is described in Swinyard et al. (1996).

\section{Analysis}

In this section we describe the analysis of the ISO spectra and the modelling of the SEDs of the hot post-AGB stars.

\subsection{ISO data analysis}

Offline processed ISO SWS01 (OLP version 10.1) and LWS01 (OLP version 10) data were retrieved from the ISO data archive. These were further processed using ISAP (ISO Spectroscopic Analysis Package) version 2.1.

\subsubsection{SWS}

The data analysis using ISAP consisted of extensive bad data removal primarily to minimize the effect of cosmic ray hits. All detectors were compared to identify possible features. For each sub-band, the flux level of the 12 detectors were shifted and brought to a mean value. They were then averaged, using median clipping to discard points that lay more than $2.5 \sigma$ from the median flux. The spectra were averaged typically to a resolution of 300, 500, 800 and 1500 for SWS01 data taken with speed 1, 2, 3 and 4 respectively (Table 2). Appropriate scaling factors were applied to the averaged spectra of each sub-band to form a continous spectrum from 2.38-45.2 $\mu$. The data of subband 3E $(27.5-29.0 \mu)$ are generally noisy and unreliable (see e.g. Hrivnak et al. 2000; Hony et al. 2002). Figures 1a-c and 2a show the SWS spectra of the hot post-AGB stars. The SWS data of IRAS 14331-6435 (Hen3-1013), IRAS 18062+2410 $($ SAO 85766$)$ and IRAS $22023+5249$ (LSIII +5224) below $7 \mu$ and that of IRAS 16206-5956 (SAO 243756), IRAS $22495+5134$ (LSIII +5142 ) below $12.5 \mu$ were noisy and have been excluded. This is also evident from the low IRAS $12 \mu$ flux ( $<5 \mathrm{Jy})$ of these stars. Identification of the infrared spectral features is based on Waters \& Molster (1999), Cox (1993), Hrivnak et al. (2000), Volk et al. (2002), Cernicharo et al. (1999) and Jørgensen et al. (2000).

\subsubsection{LWS}

Reduction of the LWS observation of IRAS 17423-1755 (Hen3-1475) consisted of extensive bad data removal using ISAP rebinning on a fixed resolution grid of $\lambda / \Delta \lambda=250$. Appropriate scaling factors were applied to the data from different LWS detectors to form a continuous spectrum (Fig. 2).
The LWS spectrum of IRAS 17423-1755 (Hen3-1475) appears featureless.

\subsubsection{Joining the SWS and LWS spectra}

Although the spectral shape is very reliable, the absolute flux calibration uncertainity is $30 \%$ for the SWS at $45 \mu$ (Schaeidt et al. 1996) and 10-15\% for the LWS at $45 \mu$ (Swinyard et al. 1998). The SWS and LWS spectra of IRAS 17423-1755 (Hen3-1475) were scaled according to their fluxes in the overlap region. The difference between the flux levels of LWS and SWS in the overlap region was smaller than $30 \%$ which is acceptable within the limits of the combined error bars. The combined SWS-LWS spectra of IRAS 17423-1755 (Hen3-1475) was used in Fig. 3.

\subsection{Spectral energy distributions (SEDs)}

To re-construct the spectral energy distributions (SEDs) of the objects, we combined the ISO data with available $U, B, V, R$, $I, J, H, K, L, L$ ' and $M$ magnitudes of the stars from literature (Table 3a). We also searched the 2MASS (2Micron All Sky Survey) Catalog within 15" of each object for their $J H K$ magnitudes and the Midcourse Space Experiment (MSX) catalog within $3^{\prime \prime}$ of each object. The 2MASS data was included only when it was found to be free from confusion flags. MSX fluxes were found only for IRAS 17460-3114 (SAO 209306; Table 3b). Infrared data $(8.7,10,11.4,12.6$ and $19.5 \mu)$ on IRAS 18062+2410 (SAO 85766; Table 3c) was obtained from Lawrence et al. (1990).

\subsubsection{Central star temperatures}

The temperatures of the central stars (Table 4) are mainly from Gauba \& Parthasarathy (2003). For IRAS 12584-4837 (Hen3-847) and IRAS 17423-1755 (Hen3-1475), we estimated the central star temperatures based on their spectral types. For the PN, IRAS 22495+5134 (LSIII +5142) we used a central star temperature of $35000 \mathrm{~K}$ (Tylenda \& Stasińska 1994). Gauba \& Parthasarathy (2003) found that the UV(IUE) spectrum of IRAS $22023+5249$ (LSIII +5224) closely resembles that of a B2-supergiant. Hence we adopted a temperature of $18500 \mathrm{~K}$ for the star, corresponding to the spectral type B2I. LTE analysis of the high resolution optical spectra of IRAS $18062+2410$ (SAO 85766) was carried out by Mooney et al. (2002) and Parthasarathy et al. (2000b). Recently, using non-LTE analysis, Ryans et al. (2003) reported an effective temperature of $20750 \mathrm{~K}$ for IRAS $18062+2410$ (SAO 85766) and $20500 \mathrm{~K}$ for IRAS 19590-1249(LSIV-12 111). In this paper, we have adopted the temperature estimates by Ryans et al. (2003).

\subsubsection{Reddening}

The interstellar extinction $\left(E(B-V)_{\text {I.S. }}\right)$ in the direction of the stars were estimated using the Diffuse Infrared Background Experiment (DIRBE)/IRAS dust maps 

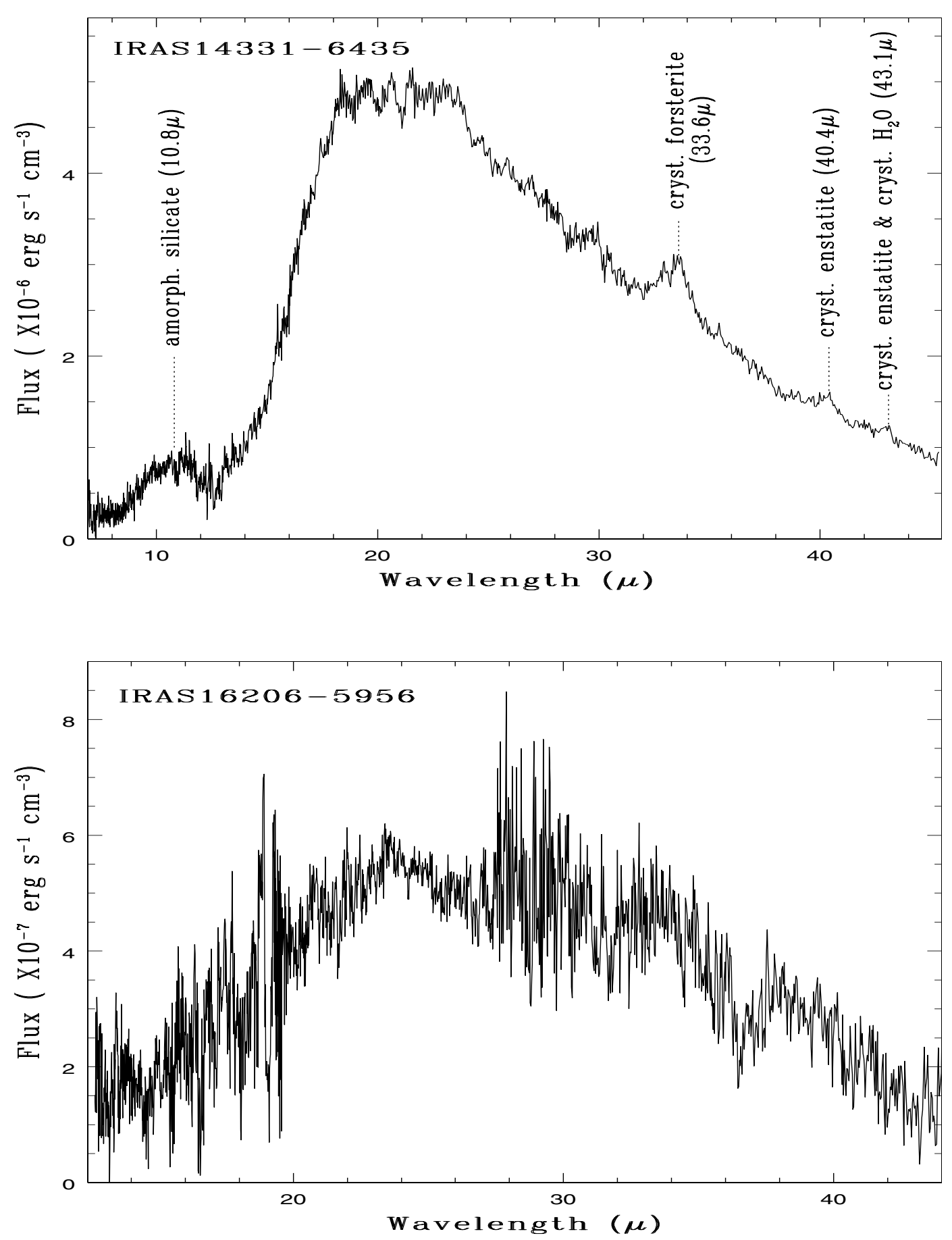

Fig. 1a. The ISO SWS spectrum of IRAS 14331-6435 (Hen3-1013) shows emission due to amorphous (10.8 $\mu$ ) and crystalline silicates and/or $\mathrm{H}_{2} \mathrm{O}(33.6 \mu, 40.4 \mu, 43.1 \mu$ ) (see e.g. Waters \& Molster 1999). ISO spectrum of IRAS 16206-5956 (SAO 243756) is noisy and only the continuum is seen here.

(Schlegel et al. 1998; Table 4). The optical spectral types of the stars are mainly from Parthasarathy et al. (2000a). The intrinsic $B-V$ values, $(B-V)_{\mathrm{o}}$, for the optical spectral types of the stars, were taken from Schmidt-Kaler (1982). We adopted $(B-V)_{\mathrm{o}}=-0.20$ for IRAS 12584-4837 (Hen3-847) and IRAS 17423-1755 (Hen3-1475) corresponding to $T_{\text {eff }}=20000 \mathrm{~K}$. For IRAS $22023+5249($ LSIII +5224$)$, we used $(B-V)_{0}=-0.16$ corresponding to B2I spectral type and for the PN, IRAS 22495+5134 (LSIII +5142), we adopted $(B-V)_{\mathrm{o}}=-0.30$ correspoding to $T_{\text {eff }}=35000 \mathrm{~K}$. Using the observed and intrinsic $B-V$ values we derived the total (interstellar plus circumstellar) extinction, $E(B-V)_{\text {total }}\left(=(B-V)_{\mathrm{obs}}-(B-V)_{\mathrm{o}}\right)$ towards these stars. Comparing $E(B-V)_{\text {total }}$ and $E(B-V)_{\text {I.S. }}$, it is evident that there is considerable circumstellar extinction in most cases. Gauba \& Parthasarathy (2003) found that the circumstellar extinction law in the UV (from $\sim 1300 \AA$ to $3200 \AA$ ) varies linearly as $\lambda^{-1}$ in the case of IRAS 13266-5551 

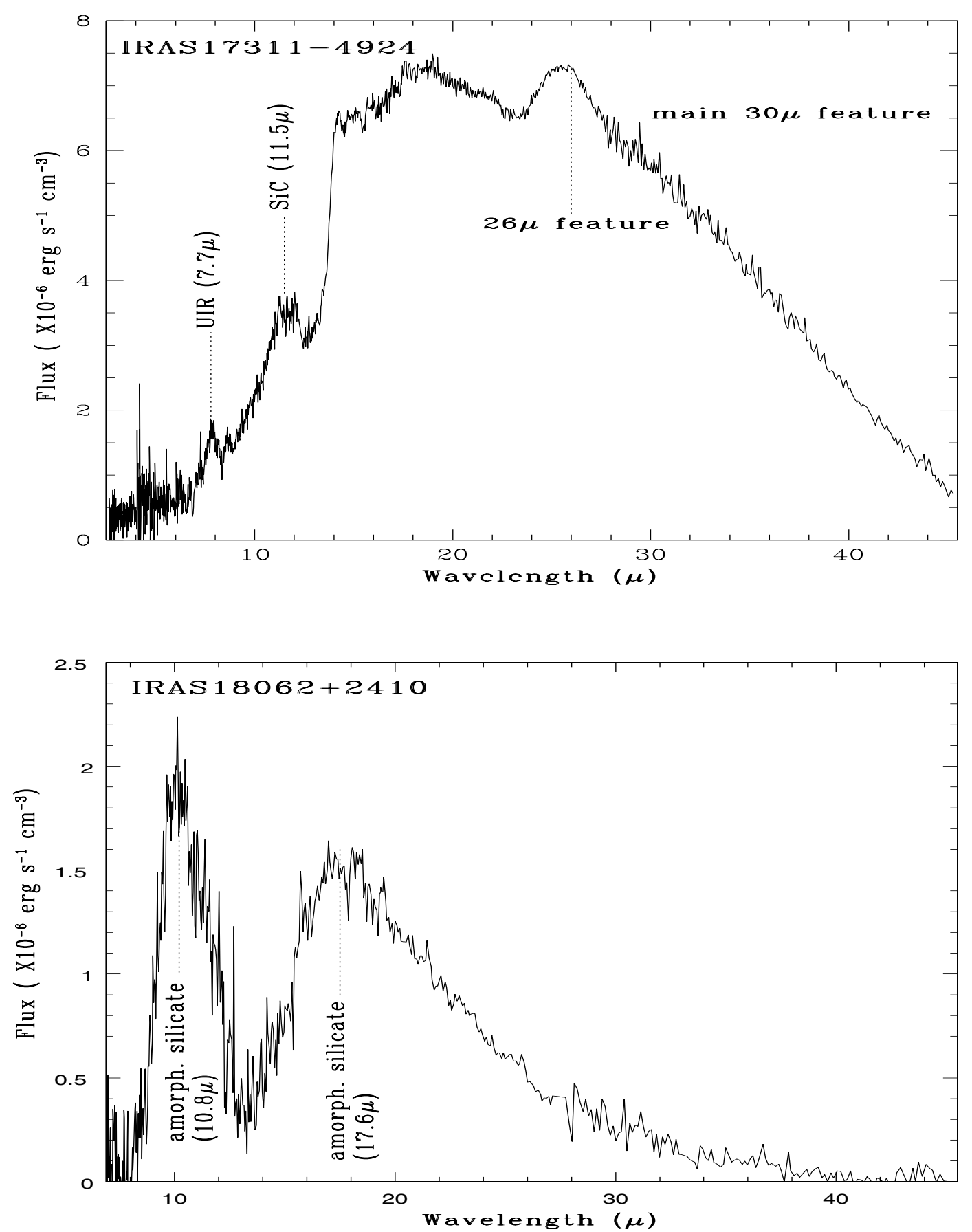

Fig. 1b. The ISO SWS spectrum of IRAS 17311-4924 (Hen3-1428) shows emission due to the UIR band at $7.7 \mu$, SiC (11.5 $\mu)$ and the " $30 \mu$ feature" (see e.g. Hrivnak et al. 2000; Volk et al. 2002). IRAS 18062+2410 (SAO 85766) shows emission due to amorphous silicates at $10.8 \mu$ and $17.6 \mu$ (see e.g. Waters \& Molster 1999).

(CPD-55 5588), IRAS 14331-6435 (Hen3-1013), IRAS 16206-5956 (SAO 243756), IRAS 17311-4924 (Hen31428), IRAS 18023-3409 (LSS 4634), IRAS 18062+2410 (SAO 85766), IRAS 18371-3159(LSE 63), IRAS 22023+5249 $($ LSIII +5224) and IRAS 22495+5134 (LSIII +5142).

Since little is known about circumstellar extinction laws, we corrected the observed optical and near infrared magnitudes of the stars for the total extinction $\left(E(B-V)_{\text {total }}\right)$ using the standard extinction laws by Rieke \& Lebofsky (1985). In particular, Rieke \& Lebofsky (1985) assume $R_{v}=3.1$, where,
$R_{v}=A_{v} / E(B-V)$. Although this is true for interstellar extinction, it may not be strictly true for circumstellar extinction obeying a $\lambda^{-1}$ law in the UV. In particular, $R_{v}$ may be different from 3.1 in the case of circumstellar extinction.

\subsubsection{Modelling the circumstellar dust shells with DUSTY code}

The use of the radiative transfer code, DUSTY (Ivezić et al. 1999) for modelling the circumstellar dust shells of hot 

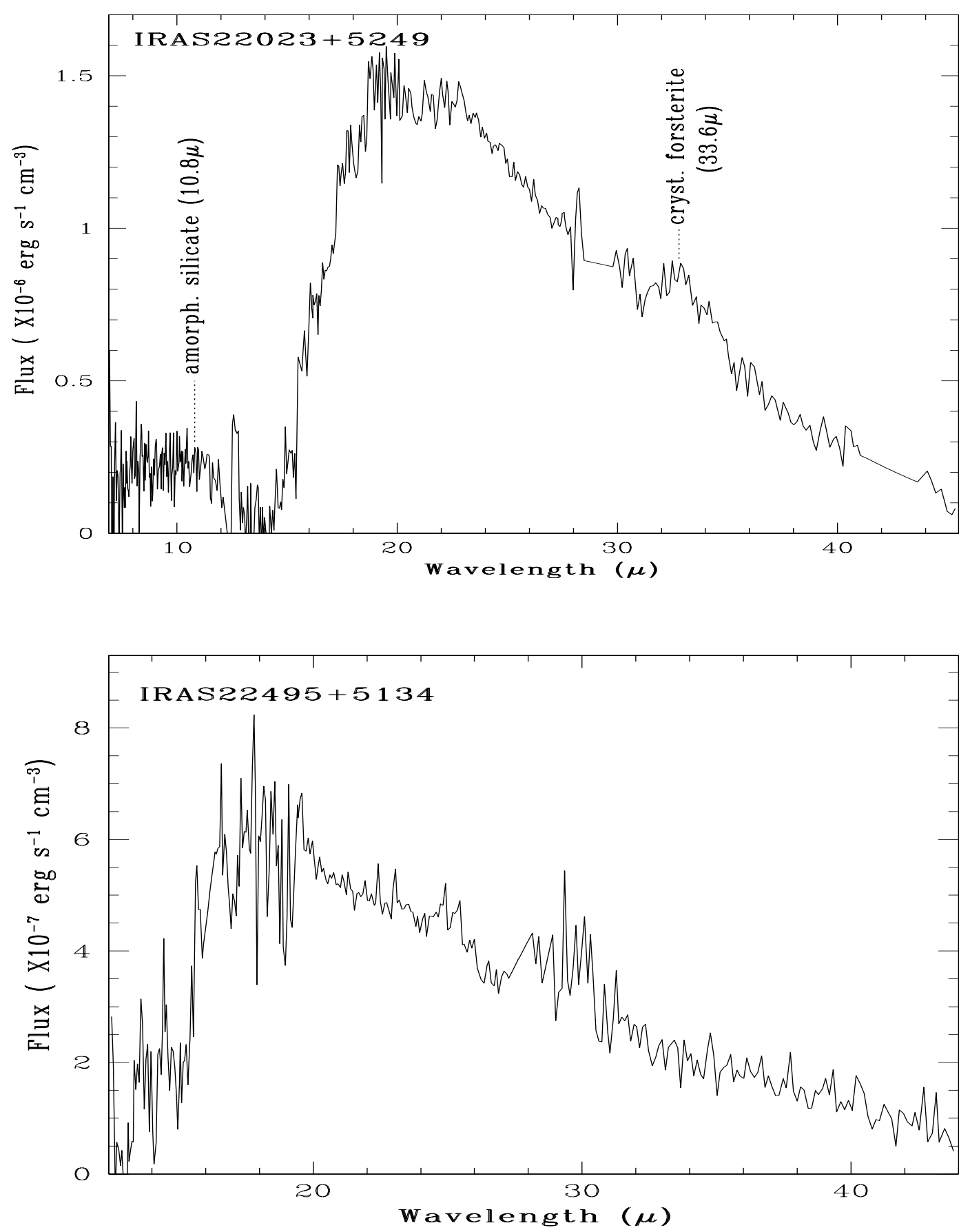

Fig. 1c. The ISO SWS spectrum of IRAS $22023+5249$ (LSIII +5224) shows emission due to amorphous $(10.8 \mu)$ and crystalline $(33.6 \mu)$ silicates (see e.g. Waters \& Molster 1999). ISO SWS spectrum of IRAS 22495+5134 (LSIII +5142) is noisy and only the continuum is seen here.

post-AGB stars was described in Gauba et al. (2003). DUSTY uses six different grain types: "warm" (Sil-Ow) and "cold" (Sil-Oc) silicates from Ossenkopf et al. (1992), silicates and graphites (Sil-Dl and grf-DL) from Draine \& Lee (1984), amorphous carbon (amC-Hn) from Hanner (1988) and $\mathrm{SiC}$ (SiC-Pg) from Pégourié (1988). The central stars were assumed to be point sources at the centers of the spherical density distributions. The SEDs of the central stars were assumed to be Planckian. The standard Mathis, Rumpl, Nordsieck (MRN) (Mathis et al. 1977) power-law was used for the grain size
( $n(a))$ distributions, i.e. $n(a) \propto a^{-q}$ for $a_{\min } \leq a \leq a_{\max }$ with $q=3.5, a(\min )=0.005 \mu$ and $a(\max )=0.25 \mu$. For each object, the dust temperature $\left(T_{\mathrm{d}}\right)$ on the inner shell boundary and the optical depth $(\tau)$ at $0.55 \mu$ ( $V$-band) were varied. We assumed an inverse square law $\left(y^{-2}\right)$ for the spherical density distribution. The shell was assumed to extend to 1000 times its inner radius. We adopted the fits for which the sum of squares of the deviations between the observed and modelled fluxes (after scaling) were a minimum. Table 4 lists the adopted input parameters. Figure 3 shows the spectral energy distribution 


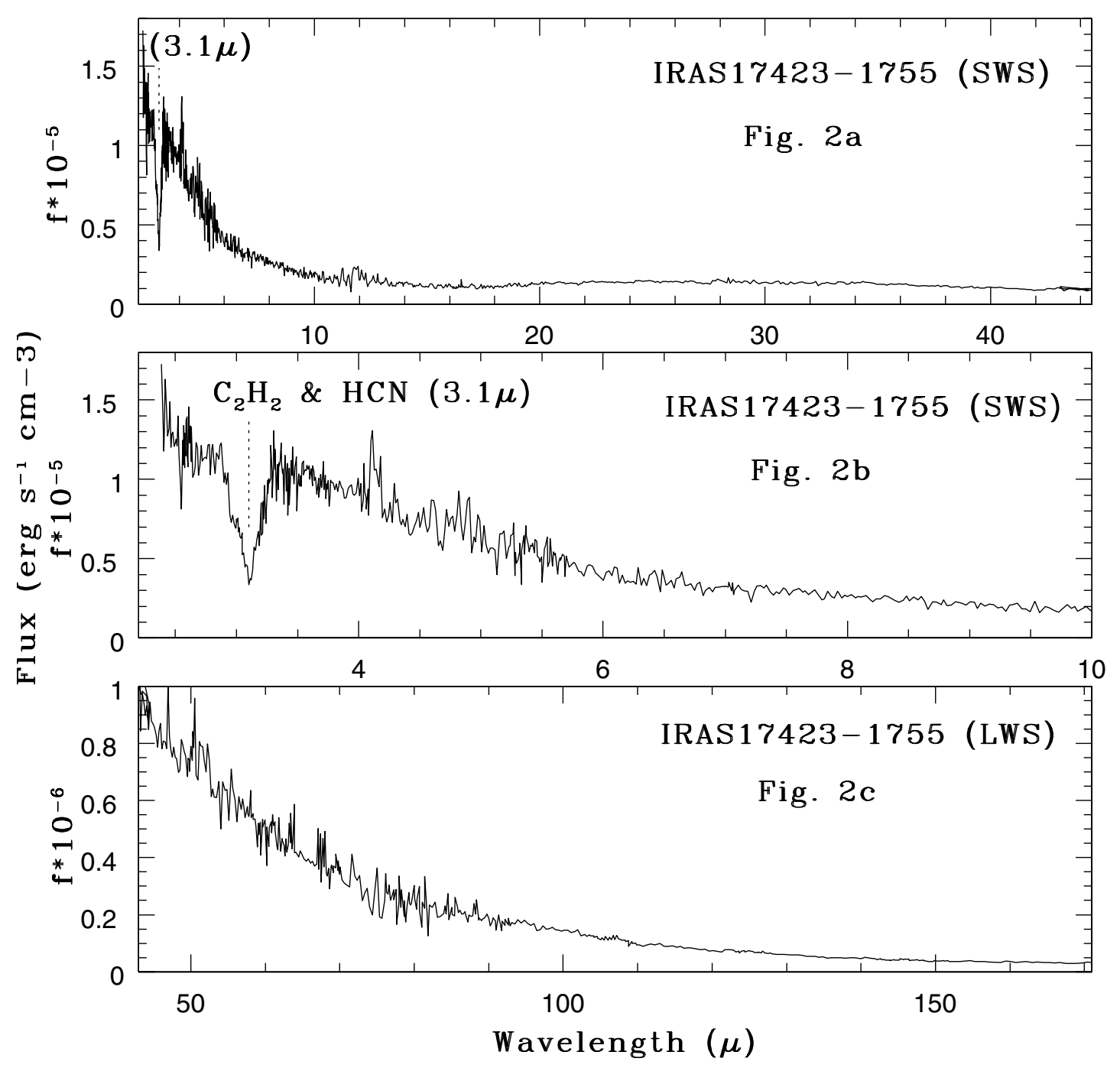

Fig. 2. ISO SWS $(2.4 \mu-44.4 \mu)$ and LWS (43 $\mu-171 \mu)$ spectra of IRAS 17423-1755 (Hen3-1475) are presented in Figs. 2a and 2c respectively. The SWS spectrum shows a broad absorption feature at $3.1 \mu$ due to $\mathrm{C}_{2} \mathrm{H}_{2}$ and/or HCN (see e.g. Cernicharo et al. 1999; Jørgensen et al. 2000). This broad absorption feature is seen clearly in Fig. $2 b$ showing the SWS spectrum of the star from $2.4 \mu-10 \mu$ only. The LWS spectrum of the star (Fig. 2c) appears featureless.

of the stars. DUSTY does not allow simultaneous modelling of warm and cold dust shells. Hence, the cold dust in the case of IRAS 12584-4837 (Hen3-847) and IRAS 17423-1755 (Hen3-1475) had to be modelled and treated independent of the warm dust around these stars.

Having fixed $T_{\mathrm{d}}$ and $\tau$, we then used the gas-dynamical mode of the DUSTY code to derive the inner radii, $r 1(\mathrm{~cm})$ where the dust temperatures $\left(T_{\mathrm{d}}\right)$ are specified and the massloss rates $(\dot{M})$. The radius scales in proportion to $L^{1 / 2}$ where $L$ is the luminosity and the code output value corresponds to $L=$ $10^{4} L_{\odot}$. The mass-loss rate scales in proportion to $L^{3 / 4}\left(r_{\mathrm{gd}} \rho_{\mathrm{s}}\right)^{1 / 2}$ where, the gas-to-dust mass ratio, $r_{\mathrm{gd}}=200$ and the dust grain density, $\rho_{\mathrm{s}}=3 \mathrm{~g} \mathrm{~cm}^{-3}$. The hot post-AGB stars discussed in this paper have a range of core-masses (Gauba \& Parthasarathy 2003). Pottasch (1992) pointed out that the white dwarf distribution is sharply peaked with a mean mass between 0.56 and $0.58 M_{\odot}$ and central stars of PNe have coremasses which show a peak at approximately $0.6 M_{\odot}$. We carried out calculations for our hot post-AGB stars with core masses of $0.565 M_{\odot}$ and $0.605 M_{\odot}$ corresponding to luminosities of $4500 L_{\odot}$ (Schönberner 1983) and $6300 L_{\odot}$ (Blöcker $1995)$ respectively. Distances $(d)$ to the stars were derived using $r 1$ and the ratio of the observed and modelled fluxes at $0.55 \mu$. $\theta(=r 1 / d)$ is the angular radii of the inner boundary of the cold circumstellar dust envelopes.

Tables 4, 5a and b list the respective values for $T_{\mathrm{d}}, r 1, d$, $\theta$ and $\dot{M}$. All calculations were carried out using the best fit parameters for the cold circumstellar dust shells.

\subsection{Notes on individual objects}

The hot post-AGB stars in this paper, except IRAS 19590-1249 (LSIV-12 111), have been described in Gauba et al. (2003) and Gauba \& Parthasarathy (2003). Here, we describe, the ISO spectra and dust shell characteristics of some of these objects.

\#IRAS 12584-4837 (Hen3-847)

It was found to be variable in the optical (Kazarovets et al. 2000; de Winter et al. 2001).The Hipparcos magnitudes at 


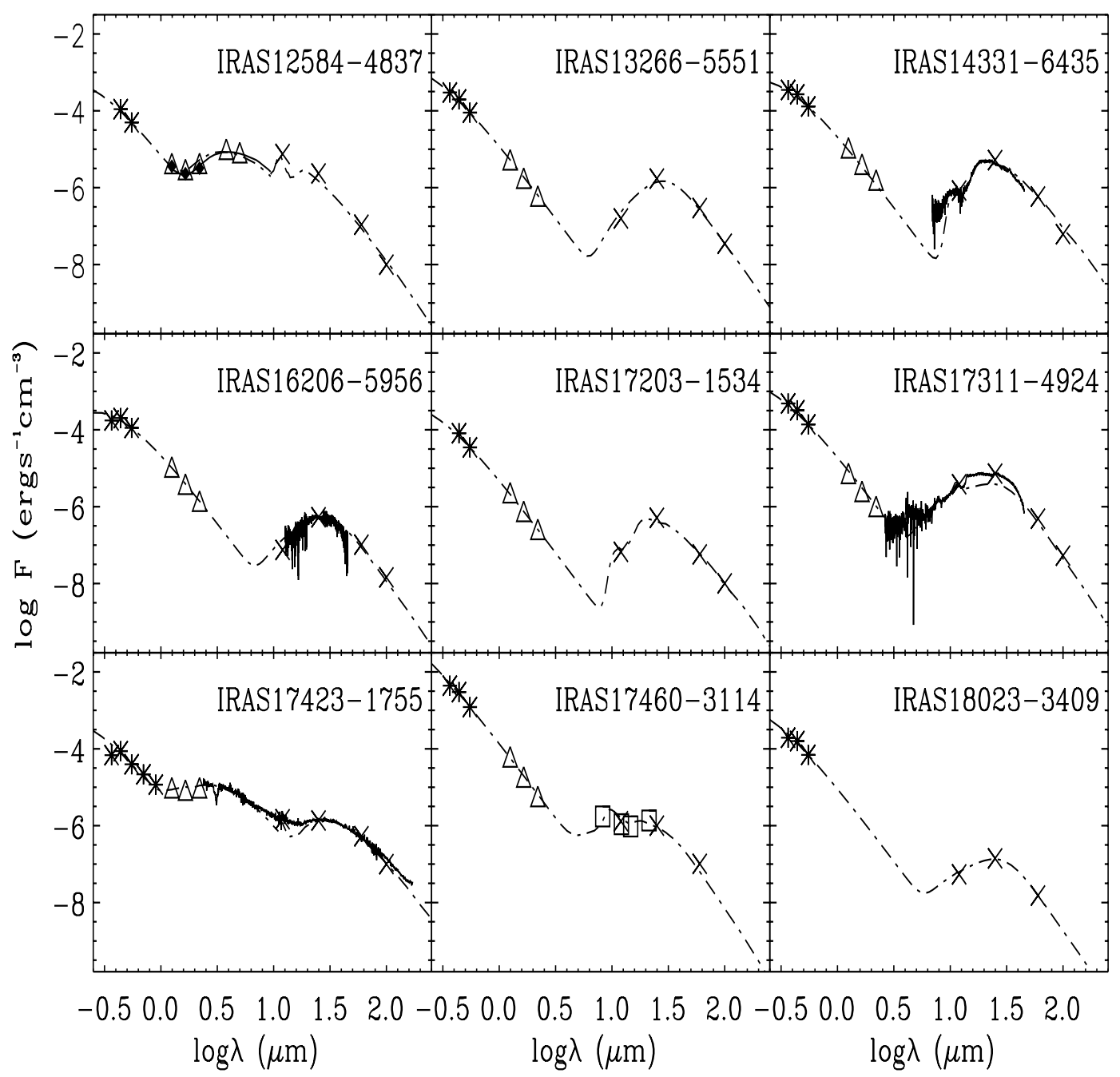

Fig. 3. Spectral energy distributions (SEDs) of the hot post-AGB stars. UBVRI data (asterisk) are plotted alongwith $J H K$ (triangle), MSX (square) and IRAS data (cross). IRAS 12584-4837 (Hen3-847), IRAS 18062+2410 (SAO 85766) and IRAS 18379-1707 (LSS 5112) are variable in $J, H$ and $K$ bands. The 2MASS, $J, H, K$ data of these three stars is represented by filled diamonds. ISO spectra of the hot post-AGB stars are plotted as solid lines. Dusty model fits are shown by dashed-dotted lines. The model fit corresponding to the $2 \mathrm{MASS} J, H, K$ data of IRAS 12584-4837 (Hen3-847) is shown by a solid line. We could not obtain a fit to the "30 $\mu$ emission feature" in IRAS 17311-4924 (Hen3-1428). The IR $(8.7,10,11.4,12.6$ and $19.5 \mu)$ and $K, L, M$ data of IRAS 18062+2410 (SAO 85766) by Lawrence et al. (1990) is indicated by diamonds. Notice the mismatch between the $K$-band flux of Lawrence et al. (1990; diamond) and García-Lario et al. (1997b; triangle) for the star. The $L$ and $M$-band fluxes also do not lie on the modelled SED of the star (see Sect. 4.3).

maximum and minimum are $10^{\mathrm{m}} .52$ and $10^{\mathrm{m}} .70$ respectively. Comparison of the $J, H, K$ magnitudes of the star from Fouque et al. (1992) and the 2MASS catalog indicate infrared variability as well. The SED of this star shows the presence of both warm and cold circumstellar dust. We have modelled the warm dust based on the data of Fouque et al. (1992) (775 K) and the 2MASS catalog $(700 \mathrm{~K})$. The presence of warm circumstellar dust may indicate ongoing post-AGB mass loss. The use of amorphous carbon grains to model the warm dust and silicate grains for the cold dust, indicates that during its evolution along the AGB, the central star may have evolved from an oxygen-rich to a carbon-rich star.

\section{\#IRAS 14331-6435 (Hen3-1013)}

The ISO SWS spectrum reveals the presence of amorphous $(10.8 \mu)$ and crystalline silicates and/or water $(33.6 \mu, 40.4 \mu$, $43.1 \mu)$ in the circumstellar environment of this star. Crystalline silicates have been detected in the dust shells around evolved oxygen-rich stars (see e.g. Waters et al. 1996; Waters \& Molster 1999). Waters et al. (1996) found that these emission features are more prominent for objects with cooler dust shells $(T<$ $300 \mathrm{~K})$. Emission from crystalline water has been reported in the $40-70 \mu$ spectrum of the Frosty Leo nebula and other cool oxygen-rich envelopes (Omont et al. 1990).

\section{\#IRAS 16206-5956 (SAO 243756)}

The ISO spectrum of this star is noisy and no features could be identified. The continuum from $12.5 \mu$ to $45.2 \mu$ was used in addition to the IRAS fluxes to better constrain the model fit to the SED.

\#IRAS 17311-4924 (Hen3-1428)

The broad "30 $\mu$ emission feature" was detected in several AGB stars and PN sources (Forrest et al. 1981; Cox 1993). More recently it was also detected in carbon-rich PPNs 


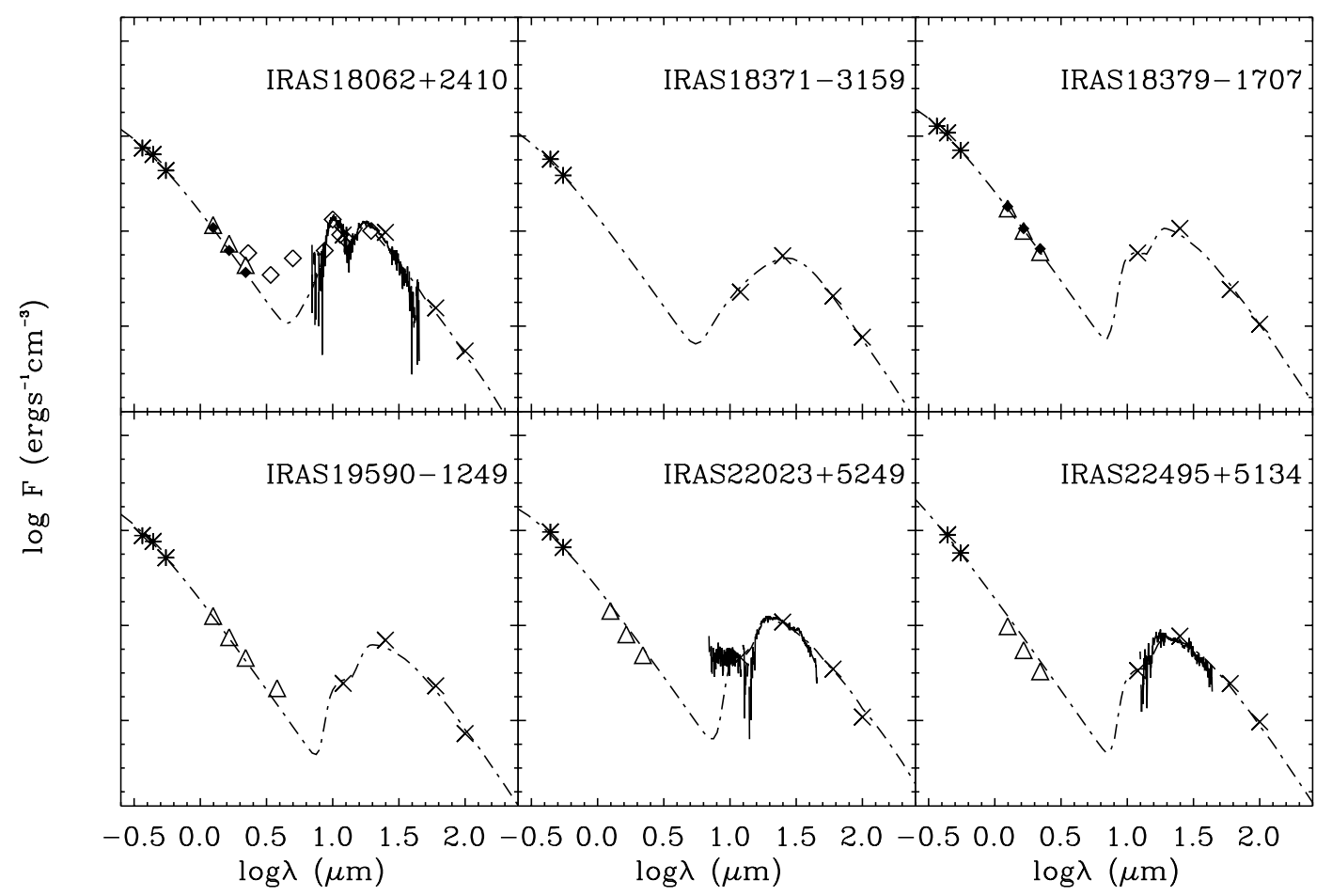

Fig. 3. continued.

possessing the $21 \mu$ emission feature (Omont et al. 1995). Hony et al. (2002) detected this feature in the ISO SWS spectrum of IRAS 17311-4924 (Hen3-1428) and a large sample of carbon-rich AGB stars (C-stars), post-AGB stars and PNe. Substructure in the $30 \mu$ feature was recognised by Szczerba et al. (1999). In the spectra of several PPNs and carbon stars, it was resolved into two components (Hrivnak et al. 2000; Volk et al. 2000, 2002), the " $26 \mu$ " and "main $30 \mu$ " feature (Fig. 1b). Goebel \& Moseley (1985) first suggested that the feature is due to magnesium sulfide (MgS). Hony et al. (2002) too identified $\mathrm{MgS}$ as the carrier of the " $26 \mu$ " and "main $30 \mu$ " emission features. However, the $30 \mu$ band is never seen in oxygen-rich sources (Forrest et al. 1979). Since the feature is seen only in carbon-rich objects, the suggestion that its carrier is a carbonaceous material continues to be appealing (Volk et al. 2002). We modelled the SED of IRAS 17311-4924 (Hen3-1428) using graphite and silicon carbide (SiC). However, we could not obtain a fit in the $30 \mu$ emission region (from $\sim 20-30 \mu$ ).

The $11.5 \mu$ band (Fig. 1b) is attributed to SiC (Treffers \& Cohen 1984) and has been detected in carbon-rich evolved objects (see e.g., Cernicharo et al. 1989). Cox (1993) pointed out that all AGB stars where a $30 \mu$ emission band is present show the $11.5 \mu$ band in emission with the notable exception of GL 3068 where it is seen in absorption. However, the reverse is not true and some caron-rich sources with a a prominent $11.5 \mu$ emission band do not show the $30 \mu$ band (V Cyg, S Cep and Y CVn). Using the DUSTY model, we obtained a good fit to the $\mathrm{SiC}$ emission.

We also detected the $7.7 \mu$ UIR feature in the ISO spectrum of this object. UIR features are commonly attributed to polyaromatic hydrocarbons (PAHs) and have been detected in other carbon-rich protoplanetary nebulae (see e.g. Beintema et al. 1996; Hrivnak et al. 2000).

\section{\#IRAS 17423-1755 (Hen3-1475)}

Gauba et al. (2003) modelled the SED of this star using DUSTY. They found a warm dust component at $1500 \mathrm{~K}$ in addition to the cold-dust at $100 \mathrm{~K}$ indicating ongoing post-AGB mass-loss. Plotting the ISO spectrum of the star $(2.38 \mu-171 \mu)$ alongwith the photometric data from Gauba et al. (2003), nearIR data from García-Lario et al. (1997b) and IRAS fluxes and using the DUSTY code, we detected a second warm dust component at $1000 \mathrm{~K}$.

The broad absorption feature at $3.1 \mu$ seen in the ISO spectrum is due to $\mathrm{C}_{2} \mathrm{H}_{2}$ and/or HCN (Ridgway et al. 1978; Cernicharo et al. 1999; Jørgensen et al. 2000). This feature is observed in carbon stars (Merrill \& Stein 1976; Noguchi et al. 1977; Groenewegen et al. 1994). The far-IR flux distribution of the star was modelled with amorphous carbon and silicon carbide confirming the carbon rich nature of the circumstellar dust shell. The LWS spectrum appears featureless.

\section{\#IRAS 18062+2410 (SAO 85766)}

High resolution optical spectra (Parthasarathy et al. 2000b; Mooney et al. 2002; Ryans et al. 2003) indicated the underabundance of carbon in this star. The ISO spectrum shows strong emission due to amorphous silicates at $10.8 \mu$ and $17.6 \mu$ in conformity with an oxygen-rich chemistry (i.e. $\mathrm{C} / \mathrm{O}<1$ ) for the central star.

The $J, H, K$ magnitudes from García-Lario et al. (1997b) and the 2MASS catalog indicate that the star is variable in the near-infrared. Optical variations of the star were detected by Arkhipova et al. (1999). The $K, L, M$ fluxes by Lawrence et al. (1990) lie above the modelled SED (Fig. 3). In particular the $K$-band flux of Lawrence et al. (1990) lies above the $K$-band 
Table 3a. Photometric data on hot post-AGB stars.

\begin{tabular}{|c|c|c|c|c|c|c|c|c|c|c|c|}
\hline IRAS & $\begin{array}{c}U \\
\mathrm{mag}\end{array}$ & $\begin{array}{c}B \\
\text { mag }\end{array}$ & $\begin{array}{c}V \\
\text { mag }\end{array}$ & $\begin{array}{c}R \\
\text { mag }\end{array}$ & $\begin{array}{c}I \\
\mathrm{mag}\end{array}$ & $\begin{array}{c}J \\
\text { mag }\end{array}$ & $\begin{array}{c}H \\
\text { mag }\end{array}$ & $\begin{array}{c}K \\
\text { mag }\end{array}$ & $\begin{array}{c}L \\
\text { mag }\end{array}$ & $\begin{array}{c}L^{\prime} \\
\mathrm{mag}\end{array}$ & $\begin{array}{c}M \\
\text { mag }\end{array}$ \\
\hline \multirow[t]{2}{*}{$12584-4837$} & & $10.65^{a}$ & $10.58^{a}$ & & & $9.99^{b}$ & $9.14^{b}$ & $7.49^{b}$ & & $4.53^{b}$ & $3.59^{b}$ \\
\hline & & & & & & $10.18^{e}$ & $9.42^{e}$ & $7.80^{e}$ & & & \\
\hline $13266-5551$ & $10.33^{c}$ & $10.99^{c}$ & $10.68^{c}$ & & & $9.96^{d}$ & $9.84^{d}$ & $9.70^{d}$ & & & \\
\hline $14331-6435$ & $11.11^{c}$ & $11.48^{c}$ & $10.90^{c}$ & & & $9.35^{d}$ & $9.03^{d}$ & $8.72^{d}$ & & & \\
\hline $16206-5956$ & $9.87^{c}$ & $10.07^{c}$ & $9.76^{c}$ & & & $9.00^{e}$ & $8.87^{e}$ & $8.71^{e}$ & & & \\
\hline $17203-1534$ & & $12.37^{a}$ & $12.02^{a}$ & & & $10.95^{e}$ & $10.81^{e}$ & $10.66^{e}$ & & & \\
\hline $17311-4924$ & $10.52^{f}$ & $11.08^{f}$ & $10.68^{f}$ & & & $9.74^{d}$ & $9.54^{d}$ & $9.19^{d}$ & & & \\
\hline $17423-1755$ & $13.57^{g}$ & $13.3^{h}$ & $12.64^{g}$ & $11.75^{g}$ & $10.91^{g}$ & $9.61^{d}$ & $8.32^{d}$ & $6.80^{d}$ & & & \\
\hline $17460-3114$ & $7.45^{c}$ & $8.17^{c}$ & $7.94^{c}$ & & & $7.32^{e}$ & $7.31^{e}$ & $7.26^{e}$ & & & \\
\hline 18023-3409 & $11.70^{c}$ & $12.01^{c}$ & $11.55^{c}$ & & & & & & & & \\
\hline \multirow[t]{2}{*}{$18062+2410$} & $10.86^{i}$ & $11.59^{i}$ & $11.54^{i}$ & & & $11.22^{d}$ & $10.97^{d}$ & $10.84^{d} ; 10.21^{j}$ & $9.61^{j}$ & & $7.30^{j}$ \\
\hline & & & & & & $11.36^{e}$ & $11.33^{e}$ & $11.23^{e}$ & & & \\
\hline $18371-3159$ & & $12.09^{a}$ & $11.98^{a}$ & & & & & & & & \\
\hline \multirow[t]{2}{*}{ 18379-1707 } & $11.94^{c}$ & $12.38^{c}$ & $11.93^{c}$ & & & $10.76^{d}$ & $10.55^{d}$ & $10.33^{d}$ & & & \\
\hline & & & & & & $10.66^{e}$ & $10.43^{e}$ & $10.16^{e}$ & & & \\
\hline 19590-1249 & $10.75^{k}$ & $11.41^{k}$ & $11.32^{k}$ & & & $11.08^{d}$ & $10.96^{d}$ & $10.78^{d}$ & & $10.34^{l}$ & \\
\hline $22023+5249$ & & $13.21^{a}$ & $12.52^{a}$ & & & $11.30^{d}$ & $11.11^{d}$ & $10.83^{d}$ & & & \\
\hline $22495+5134$ & & $12.00^{a}$ & $11.78^{a}$ & & & $11.82^{e}$ & $11.76^{e}$ & $11.57^{e}$ & & & \\
\hline
\end{tabular}

Photometry is from : ${ }^{a}$ Hog et al. (2000); ${ }^{b}$ Fouque et al. (1992); ${ }^{c}$ Reed (1998); ${ }^{d}$ García-Lario et al. (1997b); ${ }^{e}$ 2MASS; ${ }^{f}$ Kozok (1985); ${ }^{g}$ Gauba et al. (2003); ${ }^{h}$ Monet et al. (1998); ${ }^{i}$ Arkhipova et al. (1999); ${ }^{j}$ Lawrence et al. (1990); ${ }^{k}$ Arkhipova et al. (2002), ${ }^{l}$ Conlon et al. (1993a).

Table 3b. MSX data.

\begin{tabular}{c|cccc}
\hline \hline \multirow{3}{*}{ IRAS } & \multicolumn{4}{|c}{ MSX fluxes (Jansky) } \\
\cline { 2 - 5 } & Band A & Band C & Band D & Band E \\
& $8.28 \mu$ & $12.13 \mu$ & $14.65 \mu$ & $21.34 \mu$ \\
\hline $17460-3114$ & 4.0095 & 5.2726 & 6.9896 & 20.730 \\
\hline
\end{tabular}

Table 3c. Infrared data on IRAS 18062+2410 (SAO 85766).

\begin{tabular}{c|ccccc}
\hline \hline & \multicolumn{5}{|c}{ IR data in magnitudes } \\
\cline { 2 - 6 } IRAS & $8.7 \mu$ & $10 \mu$ & $11.4 \mu$ & $12.6 \mu$ & $19.5 \mu$ \\
\hline $18062+2410$ & 4.29 & 2.17 & 2.22 & 2.31 & -0.25 \\
\hline
\end{tabular}

flux estimated by García-Lario et al. (1997b). If the $K, L, M$ fluxes of the star by Lawrence et al. (1990) were not overestimated, the observed mismatch may be due to the variable nature of this object. Gauba \& Parthasarathy (2003) reported variable circumstellar extinction which in addition to stellar pulsations may be due to a dusty torus in motion around the hot central star.

Bogdanov (2000) modelled the SED of this star using a different radiative transfer code. They derived $T_{\mathrm{d}}=410 \mathrm{~K}$. This value is much higher than the dust temperature $\left(T_{\mathrm{d}}=230 \mathrm{~K}\right)$ derived by us from the model fit. Bogdanov (2000) had used the $K, L, M$ fluxes by Lawrence et al. (1990) only. Besides they did not use the ISO spectrum of the star to constrain the SED. We believe that we have a better estimate of the physical parameters of the star especially since our model gives a very good fit to the IRAS fluxes and ISO spectrum of the star.

\#IRAS 18379-1707 (LSS 5112)

The star was found to be variable in the near-infrared based on the $J, H, K$ magnitudes from García-Lario et al. (1997b) and the 2MASS catalog.

\#IRAS 19590-1249 (LSIV-12 111)

It was classified from low dispersion spectroscopy by Kilkenny \& Pauls (1990) as having a spectral type around B0. Based on an analysis of its high resolution optical spectra, McCausland et al. (1992) and Conlon et al. (1993a,b) concluded that its chemical composition and atmospheric parameters are consistent with a post-AGB evolutionary state. The presence of nebular emission lines of [NII], [OII] and [SII] in its optical spectrum suggest that it may be in the early stages of PN formation. Study of the atmospheric parameters and abundance analysis of the star have also been carried out by Mooney et al. (2002) and more recently a non-LTE analysis by Ryans et al. (2003). Based on these studies it has been confirmed that the central star of LSIV-12 111 shows severe carbon deficiency. Using silicate grain composition for the dust envelope, we obtained a good fit to the SED (Fig. 3). We estimated a dust temperature of $120 \mathrm{~K}$ which is in good agreement with the estimate of Conlon et al. (1993b) based on the $100 \mu$ IRAS flux and the color-color diagram of Jourdain de Muizon et al. (1990). Recently, Bogdanov (2003) modelled the spectral energy distribution of this star using the DUSTY code. Although they adopted different values for the extinction and the effective 
Table 4. Input physical parameters for DUSTY and the adopted reddening values.

\begin{tabular}{cccccccc}
\hline \hline IRAS & $\begin{array}{c}\text { Dust } \\
\text { type }\end{array}$ & $\begin{array}{c}E(B-V) \\
\text { total }\end{array}$ & $\begin{array}{c}E(B-V) \\
\text { I.S. }\end{array}$ & $\begin{array}{c}T_{\text {eff }} \\
(\mathrm{K})\end{array}$ & Grain types ${ }^{\dagger}$ & $\begin{array}{c}\text { Optical depth } \\
(\tau) \text { at } 0.55 \mu\end{array}$ & $\begin{array}{c}T_{\mathrm{d}} \\
(\mathrm{K})\end{array}$ \\
\hline $12584-4837^{*}$ & warm $^{1}$ & 0.27 & 0.18 & 20000 & amC-Hn & 0.26 & 775 \\
& warm $^{2}$ & 0.27 & 0.18 & 20000 & amC-Hn & 0.29 & 700 \\
& cold & 0.27 & 0.18 & 20000 & Sil-DL \& SiC & 0.29 & 200 \\
$13266-5551$ & cold & 0.51 & 0.53 & 20800 & grf-DL & 0.12 & 160 \\
$14331-6435$ & cold & 0.71 & - & 16200 & Sil-Ow & 0.50 & 130 \\
$16206-5956$ & cold & 0.29 & 0.22 & 11200 & grf-DL \& amC-Hn & 0.16 & 170 \\
$17203-1534$ & cold & 0.61 & 0.44 & 19000 & Sil-Ow & 0.15 & 117 \\
$17311-4924$ & cold & 0.66 & 0.22 & 20300 & grf-DL \& SiC-Pg & 0.22 & 250 \\
$17423-1755$ & warm & 0.86 & 0.67 & 20000 & amC-Hn & 0.16 & 1500 \\
& warm & 0.86 & 0.67 & 20000 & amC-Hn & 0.32 & 1000 \\
$17460-3114$ & cold & 0.86 & 0.67 & 20000 & SiC-Pg & 0.35 & 100 \\
$18023-3409$ & cold & 0.54 & - & 35000 & Sil-DL \& grf-DL & 0.0015 & 315 \\
$18062+2410^{*}$ & cold & 0.70 & 0.44 & 20300 & grf-DL & 0.015 & 220 \\
$18371-3159$ & cold & 0.24 & 0.11 & 20750 & Sil-Oc \& Sil-DL & 0.32 & 230 \\
$18379-1707^{*}$ & cold & 0.71 & - & 19000 & grf-DL & 0.13 & 180 \\
$19590-1249$ & cold & 0.29 & 0.20 & 20500 & Sil-Ow \& Sil-DL & 0.08 & 120 \\
$22023+5249$ & cold & 0.85 & - & 18500 & Sil-Oc \& Sil-DL & 0.29 & 120 \\
$22495+5134$ & cold & 0.52 & 0.36 & 35000 & Sil-DL & 0.011 & 125 \\
\hline
\end{tabular}

$\dagger$ The grain types used for modelling the SEDs are "warm" (Sil-Ow) and "cold" (Sil-Oc) silicates from Ossenkopf et al. (1992); silicates and graphites (Sil-Dl and grf-DL) from Draine \& Lee (1984), amorphous carbon (amC-Hn) from Hanner (1988) and SiC (SiC-Pg) from Pégourié (1988). * IRAS 12584-4837 (Hen3-847), IRAS 18062+2410 (SAO 85766) and IRAS 18379-1707 (LSS 5112) are variable in $J, H, K$ bands (see Table 3a). Hence, two separate models satisfied the warm dust component in IRAS 12584-4837 (Hen3-847). Model 1 conforms to the $J, H, K$ data from Fouque et al. (1992). Model 2 conforms to the $J$, $H, K$ data from the 2MASS catalog.

temperature of the central star, we find their derived values for the dust temperature, distance and mass loss rate to be in good agreement with our results.

\#IRAS 22023+5249 (LSIII +5224)

Amorphous $(10.8 \mu)$ and crystalline $(33.6 \mu)$ silicate features were detected in the ISO spectrum of the dust shell surrounding this star indicating the oxygen-rich nature of the central star.

\#IRAS 22495+5134 (LSIII +5142)

The ISO spectrum of this PN is noisy and spectral features could not be identified. The angular radii $(\theta)$ derived using the DUSTY code (Tables 5a and b) are in good agreement with the angular diameter of 4 " reported by Tylenda \& Stasińska (1994) and Acker et al. (1992) respectively. From the Hipparcos and Tycho Catalogues (ESA 1997), the PN has a parallax of 0.42 mas. This implies a distance of $2.38 \mathrm{kpc}$. Assuming a core mass $\left(M_{\mathrm{c}}\right)$ of $0.605 M_{\odot}$, we derived a distance of $2.17 \mathrm{kpc}$ to the PN.

\section{Discussion and conclusions}

We have modelled the circumstellar dust shells of 15 hot postAGB stars using the radiative transfer code, DUSTY and derived their dust temperatures, distances to the stars, mass loss rates and angular radii of the inner boundary of the dust envelopes (Tables 5a and b). These stars have detached dust shells (as is evident from the SEDs, Fig. 3), OB-giant or supergiant spectra and cold dust between 100-315 K, satisfying the observational properties of PPNe as defined by Kwok (1993, 2001). In addition to the cold dust, warm dust was detected in the case of IRAS 12584-4837 (Hen3-847) and IRAS 17423-1755 (Hen3-1475) indicating ongoing mass loss. From the grain types used for the model fits, we may infer the chemical composition of the circumstellar dust shells. The use of both silicate and amorphous carbon grains to model the SEDs of IRAS 12584-4837 (Hen3-847) and IRAS 174603114 (SAO 209306) suggests that the central stars in these two cases may have undergone a recent change from an oxygenrich to a carbon-rich chemistry. Such hot post-AGB stars may evolve into the [WC] central stars of PNe. Recently, Waters et al. (1998) detected carbon-rich PAH features in the nearinfrared and crystalline silicates in the far-infrared ISO spectra of two PNe with [WC] central stars, BD+303639 and He2-113.

Observational evidence (e.g. Chu et al. 1991) suggests that three winds are involved in stripping the outer envelope of the AGB star on its way to becoming a PN (Marten et al. 1993; Frank 1994): the spherically symmetric AGB wind (e.g. Habing \& Blommaert 1993) when the star loses mass at rates of $10^{-7}-10^{-6} M_{\odot} \mathrm{yr}^{-1}$ with a wind velocity of $\sim 10 \mathrm{~km} \mathrm{~s}^{-1}$; the superwind phase when the mass loss is thought to increase dramatically at the end of the AGB, upto $10^{-5}-10^{-3} M_{\odot} \mathrm{yr}^{-1}$, still with a wind velocity of $\sim 10 \mathrm{~km} \mathrm{~s}^{-1}$; once the superwind 
Table 5a. Derived stellar and dust envelope parameters for $M_{\mathrm{c}}=0.565 M_{\odot}$.

\begin{tabular}{ccccccc}
\hline \hline IRAS & $\begin{array}{c}r 1 \\
(\mathrm{~cm})\end{array}$ & $\begin{array}{c}d \\
(\mathrm{kpc})\end{array}$ & $\begin{array}{c}\theta \\
\left({ }^{\prime \prime}\right)\end{array}$ & $\begin{array}{c}\dot{M} \\
M_{\odot} \mathrm{yr}^{-1}\end{array}$ & $\begin{array}{c}\mu \\
\mathrm{mas} \mathrm{yr}^{-1}\end{array}$ & $\begin{array}{c}V_{\mathrm{T}} \\
\mathrm{km} \mathrm{s}^{-1}\end{array}$ \\
\hline $12584-4837$ & $2.03 \times 10^{16}$ & 2.85 & 0.47 & $1.34 \times 10^{-5}$ & 9.96 & 134.89 \\
$13266-5551$ & $3.92 \times 10^{16}$ & 2.05 & 1.28 & $8.08 \times 10^{-6}$ & 8.22 & 79.52 \\
$14331-6435$ & $5.10 \times 10^{16}$ & 2.10 & 1.62 & $3.27 \times 10^{-5}$ & 6.75 & 66.66 \\
$16206-5956$ & $2.91 \times 10^{16}$ & 3.24 & 0.60 & $7.14 \times 10^{-6}$ & 3.96 & 60.55 \\
$17203-1534$ & $5.25 \times 10^{16}$ & 3.70 & 0.95 & $1.35 \times 10^{-5}$ & 9.90 & 173.80 \\
$17311-4924$ & $1.72 \times 10^{16}$ & 1.66 & 0.70 & $8.24 \times 10^{-6}$ & 4.75 & 37.52 \\
$17423-1755$ & $2.98 \times 10^{17}$ & 3.15 & 6.39 & $5.30 \times 10^{-5}$ & & \\
$17460-3114$ & $9.93 \times 10^{15}$ & 0.30 & 2.19 & $1.86 \times 10^{-7}$ & 3.90 & 5.55 \\
$18023-3409$ & $3.12 \times 10^{16}$ & 2.46 & 0.58 & $1.25 \times 10^{-6}$ & 13.93 & 161.04 \\
$18062+2410$ & $9.13 \times 10^{15}$ & 4.48 & 0.14 & $1.03 \times 10^{-5}$ & 12.59 & 271.26 \\
$18371-3159$ & $3.14 \times 10^{16}$ & 5.04 & 0.41 & $7.69 \times 10^{-6}$ & 7.94 & 188.18 \\
$18379-1707$ & $3.00 \times 10^{16}$ & 3.10 & 0.64 & $1.12 \times 10^{-5}$ & & \\
$19590-1249$ & $4.80 \times 10^{16}$ & 3.93 & 0.82 & $9.89 \times 10^{-6}$ & 7.56 & 143.34 \\
$22023+5249$ & $4.62 \times 10^{16}$ & 3.33 & 0.93 & $2.09 \times 10^{-5}$ & 4.96 & 78.37 \\
$22495+5134$ & $5.59 \times 10^{16}$ & 1.83 & 2.04 & $3.05 \times 10^{-6}$ & 2.97 & 25.60 \\
\hline
\end{tabular}

Table 5b. Derived stellar and dust envelope parameters for $M_{\mathrm{c}}=0.605 M_{\odot}$.

\begin{tabular}{ccccccc}
\hline \hline IRAS & $\begin{array}{c}r 1 \\
(\mathrm{~cm})\end{array}$ & $\begin{array}{c}d \\
(\mathrm{kpc})\end{array}$ & $\begin{array}{c}\theta \\
\left({ }^{\prime \prime}\right)\end{array}$ & $\begin{array}{c}\dot{M} \\
M_{\odot} \mathrm{yr}^{-1}\end{array}$ & $\begin{array}{c}\mu \\
\mathrm{mas} \mathrm{yr}^{-1}\end{array}$ & $\begin{array}{c}V_{\mathrm{T}} \\
\mathrm{km} \mathrm{s}^{-1}\end{array}$ \\
\hline $12584-4837$ & $2.40 \times 10^{16}$ & 3.37 & 0.47 & $1.73 \times 10^{-5}$ & 9.96 & 157.37 \\
$13266-5551$ & $4.63 \times 10^{16}$ & 2.42 & 1.28 & $1.04 \times 10^{-5}$ & 8.22 & 95.03 \\
$14331-6435$ & $6.03 \times 10^{16}$ & 2.49 & 1.62 & $4.22 \times 10^{-5}$ & 6.75 & 80.00 \\
$16206-5956$ & $3.44 \times 10^{16}$ & 3.83 & 0.60 & $9.22 \times 10^{-6}$ & 3.96 & 72.19 \\
$17203-1534$ & $6.21 \times 10^{16}$ & 4.38 & 0.95 & $1.74 \times 10^{-5}$ & 9.90 & 204.03 \\
$17311-4924$ & $2.03 \times 10^{16}$ & 1.96 & 0.70 & $1.06 \times 10^{-5}$ & 4.75 & 44.15 \\
$17423-1755$ & $3.52 \times 10^{17}$ & 3.72 & 6.39 & $6.84 \times 10^{-5}$ & & \\
$17460-3114$ & $1.17 \times 10^{16}$ & 0.36 & 2.19 & $2.40 \times 10^{-7}$ & 3.90 & 6.65 \\
$18023-3409$ & $2.48 \times 10^{16}$ & 2.92 & 0.58 & $1.62 \times 10^{-6}$ & 13.93 & 194.20 \\
$18062+2410$ & $1.08 \times 10^{16}$ & 5.29 & 0.14 & $1.33 \times 10^{-5}$ & 12.59 & 314.09 \\
$18371-3159$ & $3.71 \times 10^{16}$ & 5.95 & 0.41 & $9.93 \times 10^{-6}$ & 7.94 & 221.38 \\
$18379-1707$ & $3.55 \times 10^{16}$ & 3.67 & 0.64 & $1.45 \times 10^{-5}$ & & \\
$19590-1249$ & $5.67 \times 10^{16}$ & 4.64 & 0.82 & $1.28 \times 10^{-5}$ & 7.56 & 170.64 \\
$22023+5249$ & $5.46 \times 10^{16}$ & 3.94 & 0.93 & $2.70 \times 10^{-5}$ & 4.96 & 94.04 \\
$22495+5134$ & $6.61 \times 10^{16}$ & 2.17 & 2.04 & $3.94 \times 10^{-6}$ & 2.97 & 30.60 \\
\hline
\end{tabular}

exhausts most of the AGB star's envelope, a fast wind with mass loss rate of $10^{-8} M_{\odot} \mathrm{yr}^{-1}$ and velocity of $\sim 1000 \mathrm{~km} \mathrm{~s}^{-1}$ develops at some point during the PPN phase. Velocities of $1000 \mathrm{~km} \mathrm{~s}^{-1}$ and mass loss rates of $\sim 10^{-8} M_{\odot} \mathrm{yr}^{-1}$ have been observed in the central stars of PNe (e.g. Gauba et al. 2001). For our hot post-AGB stars, we derived mass loss rates of $10^{-5}-10^{-6} M_{\odot} \mathrm{yr}^{-1}$. The mass loss rates $(\dot{M})$ scale with the gas-to-dust mass ratio $\left(r_{\mathrm{gd}}\right)$. We have adopted $r_{\mathrm{gd}}=200$. For carbon-rich AGB and post-AGB stars values between 200 and 250 are often used (e.g. Jura 1986; Meixner et al. 1997). For the cool (F3Ib) post-AGB star, HD 161796 (Parthasarathy \& Pottasch 1986) with an oxygen-rich circumstellar environment, Hoogzaad et al. (2002) estimated $r_{\text {gd }}=270$. Furthermore, our models assume that the dust density distribution falls off as $y^{-2}$ in the entire circumstellar dust shell. Such an assumption would break down in the case of episodic mass loss (Olofsson et al. 1990). e.g. episodic mass loss may have been responsible for the rapid evolution (30-40 years) of IRAS 171195926 (Hen3-1357) and IRAS 18062+2410 (SAO 85766) from B-type post-AGB supergiants to young PNe (Parthasarathy et al. 1993c, 1995; Bobrowsky et al. 1998; Parthasarathy et al. 2000b).

The proper motions $(\mu)$ of the stars from the Tycho- 2 Catalogue (Hog et al. 2000) have been listed in Tables 5a and $b$. Using the derived distances $(d)$ in conjunction with the proper motions we estimated the component of the stellar space velocities of the targets tangent to the line of sight $\left(V_{\mathrm{T}}\right)$. For IRAS 17203-1534, IRAS 18062+2410 (SAO 85766) and IRAS 18371-3159 (LSE63), the large $V_{\mathrm{T}}$ values (Tables 5a and b) imply very high space velocities $\left(V_{\mathrm{s}}=\left(V_{\mathrm{T}}^{2}+V_{\mathrm{r}}^{2}\right)^{1 / 2}\right.$; 
Table 6. Predicted and observed $(V-J)$ colors.

\begin{tabular}{ccccccc}
\hline \hline IRAS & $A_{\mathrm{V}}=3.1 \times E(B-V)_{\text {total }}$ & $A_{\mathrm{J}}=0.28 \times A_{\mathrm{V}}$ & $(V-J)_{\mathrm{o}}$ & $(V-J)_{\text {predicted }}$ & $(V-J)_{\text {obs }}$ & $\Delta(V-J)$ \\
\hline $12584-4837$ & 0.84 & 0.23 & - & - & $0.59^{a}$ & - \\
& & & & & $0.40^{b}$ & - \\
$13266-5551$ & 1.58 & 0.44 & -0.47 & 0.67 & 0.72 & -0.05 \\
$14331-6435$ & 2.20 & 0.62 & -0.37 & 1.21 & 1.55 & -0.34 \\
$16206-5956$ & 0.90 & 0.25 & 0.02 & 0.67 & - & - \\
$17203-1534$ & 1.89 & 0.53 & -0.47 & 0.89 & 1.07 & -0.18 \\
$17311-4924$ & 2.05 & 0.57 & -0.47 & 1.01 & 0.94 & 0.07 \\
$17423-1755$ & 2.67 & 0.75 & - & - & 3.03 & - \\
$17460-3114$ & 1.67 & 0.47 & -0.57 & 0.63 & 0.62 & 0.01 \\
$18023-3409$ & 2.17 & 0.61 & -0.47 & & - & - \\
$18062+2410$ & 0.74 & 0.21 & -0.47 & 0.06 & $0.32^{c}$ & -0.26 \\
$18371-3159$ & & & & & $0.18^{b}$ & -0.12 \\
$18379-1707$ & 0.93 & 0.26 & -0.47 & 0.20 & - & - \\
$19590-1249$ & 2.20 & 0.62 & -0.47 & 1.11 & $1.17^{c}$ & -0.06 \\
$22023+5249$ & & & & & $1.27^{b}$ & -1.16 \\
$22495+5134$ & 2.63 & 0.25 & -0.47 & 0.18 & 0.24 & -0.06 \\
\hline
\end{tabular}

Based on $J, H, K$ data from ${ }^{a}$ Fouque et al. (1992); ${ }^{b}$ 2MASS and ${ }^{c}$ García-Lario et al. (1997b).

where $V_{\mathrm{r}}$ is the radial velocity of a star), close to the escape velocity from the Galaxy of $290 \mathrm{~km} \mathrm{~s}^{-1}$ near the Sun. Mooney et al. (2002) estimated a distance of $8.1 \mathrm{kpc}$ to IRAS $18062+2410($ SAO 85766) which is much greater than our estimate of $\sim 5 \mathrm{kpc}$. Such a large distance, if correct, would imply a still higher space velocity. We believe our distance estimates to be closer to the actual values for these stars. However, the assumption of spherical density distributions in our models, may be an over simplification for some of these objects. e.g. IRAS 17423-1755 (Hen3-1475) has IRAS colors moderately close to those of HD 233517. HD 233517 is unresolved and may have a disk instead of a spherical outflow (see e.g. Jura 2003; Fisher et al. 2003). The predicted angular sizes of the inner radii of the dust shells (Tables $5 a$ and $b$ ) suggests that these objects should be easily resolvable in the mid-IR images with large ground based telescopes. Imaging of these objects in the IR would serve to test the basic assumptions such as those of spherical symmetry for our models.

In Table 6 we have compared the predicted and observed $(V-J)$ values for these stars $\left(\Delta(V-J)=(V-J)_{\text {predicted }}-(V-\right.$ $\left.J)_{\text {obs }}\right)$ where, $(V-J)_{\text {predicted }}=(V-J)_{\mathrm{o}}+A_{V}-A_{J}$. The intrinsic $(V-J)$ colors $\left((V-J)_{\mathrm{o}}\right)$, for the spectral types of the stars are from Ducati et al. (2001). For stars with emission lines in their optical spectra, IRAS 12584-4837 (Hen3-847), IRAS 174231755 (Hen3-1475), IRAS 22023+5249 (LSIII+5224) and for the PN, IRAS 22495+5134 (LSIII+5142), $(V-J)_{\text {o }}$ could not be assumed. In the case of IRAS 14331-6435 (Hen31013), IRAS 17203-1534, IRAS 18062+2410 (SAO 85766) and IRAS 18379-1707 (LSS 5112), we find significant differences between the values of $(V-J)_{\text {predicted }}$ and $(V-J)_{\text {obs }}$. On first sight, this would then raise a suspicion about the adopted $E(B-V)_{\text {total }}$ values. However, we would like to point out that the $V$ and $J$ magnitudes of these stars have not been recorded simultaneously. Many of these stars are variable as evidenced from the $J, H, K$ data on IRAS 12584-4837 (Hen3847), IRAS $18062+2410$ (SAO 85766) and IRAS 18379-1707 (LSS 5112). The $V$ and $J$ magnitudes may have be recorded at different epochs of the variability cycle and hence it may not be suitable to compare $(V-J)_{\text {predicted }}$ and $(V-J)_{\text {obs }}$.

We also studied the ISO spectra of 7 hot post-AGB stars, IRAS 14331-6435 (Hen3-1013), IRAS 162065956 (SAO 243756), IRAS 17311-4924 (Hen3-1428), IRAS 17423-1755 (Hen3-1475), IRAS 18062+2410 (SAO 85766), IRAS 22023+5249 (LSIII +5224) and IRAS 22495+5134 (LSIII +5142). A weak amorphous silicate feature $(10.8 \mu)$ alongwith crystalline silicate features was found in the dust shells of IRAS 14331-6435 (Hen3-1013) and IRAS $22023+5249$ (LSIII +5224 ). The $17.6 \mu$ amorphous silicate feature was missing in these two stars. The post-AGB star IRAS 18062+2410 (SAO 85766) did not show evidence for the presence of crystalline silicates but strong amorphous silicate features at $10.8 \mu$ and $17.6 \mu$ were detected. Volk \& Kwok (1989) predict that at dust temperatures of typically a few $100 \mathrm{~K}$ for post-AGB stars, the spectrum should increase from 8 to $23 \mu$ and the $10.8 \mu$ and $17.6 \mu$ silicate features should cease to be observable. This appears to be consistent with the observed spectral features and the dust temperatures of $230 \mathrm{~K}, 130 \mathrm{~K}$ and $120 \mathrm{~K}$ for IRAS $18062+2410$ (SAO 85766), IRAS 14331-6435 (Hen3-1013) and IRAS 22023+5249 $($ LSIII +5224$)$ respectively from our model fits. The presence of silicate features in these stars indicates the O-rich nature of the central stars. The formation of crystalline silicates in the circumstellar shells of post-AGB stars is still not well understood (see e.g., Waters et al. 1996). In contrast, PAH emission at $7.7 \mu$, the "26 $\mu$ " and "main $30 \mu$ " features and $11.5 \mu$ SiC emission in IRAS 17311-4924 (Hen3-1428), are typical 
of circumstellar dust shells around carbon-rich post-AGB stars. However, the $21 \mu$ emission feature detected in several carbon-rich PPNe (Hrivnak et al. 2000) was notably absent in the ISO spectrum of IRAS 17311-4924 (Hen3-1428). Volk et al. (2002) pointed out that although all sources with the $21 \mu$ emission feature also display the " $26 \mu$ " and "main $30 \mu$ " features, the converse is not true. The hot post-AGB star, IRAS $01005+7910$ (Klochkova et al. 2002) which showed the "26" and "main $30 \mu$ " emission also did not show the $21 \mu$ emission feature (Hrivnak et al. 2000). It may be that the dust grains responsible for the $21 \mu$ emission are destroyed as the central star evolves towards hotter temperatures. The broad absorption feature at 3.1 $\mu$ in IRAS 17423-1755 (Hen3-1475) attributed to $\mathrm{C}_{2} \mathrm{H}_{2}$ and/or $\mathrm{HCN}$ indicates that the central star may be carbon-rich.

Acknowledgements. We are grateful to the referee for helpful comments. G.G. would also like to thank Luciano Rezzolla and John Miller of the Astrophysics sector at SISSA, Trieste, Italy for providing computer facilities during her stay in Italy. The ISO Spectral Analysis Package (ISAP) is a joint development by the LWS and SWS Instrument Teams and Data Centers. Contributing institutes are CESR, IAS, IPAC, MPE, RAL and SRON.

\section{References}

Acker, A., Ochsenbein, F., Stenholm, B., et al. 1992, Strasbourg-ESO Catalogue of Galactic Planetary Nebulae (ESO publication)

Arkhipova, V. P., Ikonnikova, N. P., Noskova, R. I., et al. 1999, Astron. Lett., 25, 25

Arkhipova, V. P., Ikonnikova, N. P., Noskova, R. I., \& Komissarova, G. V. 2002, Astron. Lett., 28, 257

Beintema, D. A., van den Ancker, M. E., Molster, F. J., et al. 1996, A\&A, 315, L369

Blöcker, T. 1995, A\&A, 299, 755

Bobrowsky, M., Sahu, K. C., Parthasarathy, M., \& García-Lario, P. 1998, Nature, 392, 469

Bogdanov, M. B. 2000, Astron. Rep., 44, 685

Bogdanov, M. B. 2002, Astron. Lett., 28, 617

Bogdanov, M. B. 2003, Astron. Rep., 47, 480

Cernicharo, J., Gottlieb, C. A., Guélin, M., et al. 1989, ApJ, 341, L25

Cernicharo, J., Yamamura, I., González-Alfonso, E., et al. 1999, ApJ, 526, L41

Chu, Y., Manchado, A., Jacoby, G. H., \& Kwitter, K. B. 1991, ApJ, 376,150

Clegg, P. E., Ade, P. A. R., Armand, C., et al. 1996, A\&A, 315, L38

Conlon, E. S., Dufton, P. L., Keenan, F. P., McCausland, R. J. H., \& Little, J. E. 1993a, A\&A, 272, 243

Conlon, E. S., Dufton, P. L., McCausland, R. J. H., \& Keenan, F. P. 1993b, ApJ, 408, 593

Cox, P. 1993, ASP Conf. Ser., 41, 163 (Sun Kwok)

de Graauw, Th., Haser, L. N., Beintema, D. A., et al. 1996, A\&A, 315, L49

de Winter, D., van den Ancker, M. E., Maira, A., et al. 2001, A\&A, 380,609

Draine, B. T., \& Lee, H. M. 1984, ApJ, 285, 89

Ducati, J. R., Bevilacqua, C. M., Rembold, S. B., \& Ribeiro, D. 2001, ApJ, 558, 309

Fisher, R. S., Telesco, C. M., Pina, R. K., \& Knacke, R. F. 2003, 586, L91

Forrest, W. J., McCarthy, J. F., \& Houck, J. R. 1979, ApJ, 233, 611

Forrest, W. J., Houck, J. R., \& McCarthy, J. F. 1981, ApJ, 248, 195
Fouque, P., Le Bertre, T., Epchtein, N., et al. 1992, A\&AS, 93, 151

Frank, A. 1994, AJ, 107, 261

García-Lario, P., Parthasarathy, M., de Martino, D., et al. 1997a, A\&A, 326, 1103

García-Lario, P., Manchado, A., Pych, W., \& Pottasch, S. R. 1997b, A\&AS, 126, 479

Gauba, G., Parthasarathy, M., Nakada, Y., \& Fujii, T. 2001, A\&A, 373 , 572

Gauba, G., Parthasarathy, M., Kumar, B., Yadav, R. K. S., \& Sagar, R. 2003, A\&A, 404, 305

Gauba, G., \& Parthasarathy, M. 2003, A\&A, 407, 1007

Goebel, J. H., \& Moseley, S. H. 1985, ApJ, 290, L35

Groenewegen, M. A. T., de Jong, T., \& Geballe, T. R. 1994, A\&A, 287, 163

Habing, H. J., \& Blommaert, J. A. D. L. 1993, in IAU Symp. 155, 243, ed. A. Acker, \& R. Weinberger

Hanner, M. S. 1988, NASA Conf. Pub., 3004, 22

Hog, E., Fabricius, C., Makarov, V. V., et al. 2000, A\&A, 355, L27

Hony, S., Waters, L. B. F. M., \& Tielens, A. G. G. M. 2002, A\&A, 390,533

Hony, S., Tielens, A. G. G. M., Waters, L. B. F. M., \& de Koter, A. 2003, A\&A, 402, 211

Hoogzaad, S. N., Molster, F. J., Dominik, C., et al. 2002, A\&A, 389, 547

Hrivnak, B. J., Volk, K., \& Kwok, S. 2000, ApJ, 535, 275

Iben, I., Jr., Renzini, A. 1983, ARA\&A, 21, 271

Ivezić, Z., Nenkova, M., \& Elitzur, M. 1999, User Manual for DUSTY, University of Kentucky Internal Report

Jørgensen, U. G., Hron, J., \& Loidl, R. 2000, A\&A, 356, 253

Jourdain de Muizon, N., Cox, P., \& Lequeux, J. 1990, A\&A, 83, 337

Jura, M. 1986, ApJ, 303, 327

Jura, M. 2003, ApJ, 582, 1032

Kazarovets, E. V., Samus, N. N, \& Durlevich, O. V. 2000, IBVS. 4870, $1 \mathrm{~K}$

Kessler, M. F., Steinz, J. A., Anderegg, M. E., et al. 1996, A\&A, 315, L27

Kilkenny, D., \& Pauls, L. 1990, MNRAS, 244, 133

Klochkova, V. G., Yushkin, M. V., Miroshnichenko, A. S., et al. 2002, A\&A, 392, 143

Kozok, J. R. 1985, A\&AS, 61, 387

Kwok, S. 1993, ARA\&A, 31, 63

Kwok, S. 2001, in Post-AGB Objects as a Phase of Stellar Evolution, Ap\&SS, 265, 3, ed. R. Szczerba, \& S. K. Górny (Boston, Dordrecht, London: Kluwer Academic Publishers)

Lawrence, G., Jones, T. J., \& Gehrz, R. D. 1990, AJ, 99, 1232

Likkel, L., Forveille, T., Omont, A., \& Morris, M. 1991, A\&A, 246, 153

Marten, H., Gesicki, K., \& Szczerba, R. 1993, in IAU Symp., 155, 315, ed. A. Acker, \& R. Weinberger

Mathis, J. S., Rumpl, W., \& Nordsieck, K. H. 1977, ApJ, 17, 25

Meixner, M., Skinner, C. J., Graham, J. R., et al. 1997, ApJ, 482, 897

Merrill, K. M., \& Stein, W. A. 1976, PASP, 88, 285

Monet, D., Bird, A., Canzian, B., et al. 1998, USNO-A V2.0, A Catalog of Astrometric Standards

Mooney, C. J., Rolleston, W. R. J., Keenan, F. P., et al. 2002, MNRAS, 337,851

Noguchi, K., Maihara, T., Okuda, H., et al. 1977, PASJ, 29, 511

Olofsson, H., Carlstrom, U., Eriksson, K., Gustafsson, B., \& Willson, L. A. 1990, A\&A, 230, L13

Omont, A., Forveille, T., \& Moseley, S. H. 1990, ApJ, 355, L27

Omont, A., Moseley, S. H., Cox, P., et al. 1995, ApJ, 454, 819

Ossenkopf, V., Henning, Th., \& Mathis, J. S. 1992, A\&A, 261, 567

Parthasarathy, M., \& Pottasch, S. R. 1986, A\&A, 154, L16 
Parthasarathy, M., \& Pottasch, S. R. 1989, A\&A, 225, 521

Parthasarathy, M. 1993a, ApJ, 414, L109

Parthasarathy, M. 1993b, in Luminous High-Latitude Stars, ed. D. D Sasselov, ASP Conf. Ser., 45, 173

Parthasarathy, M., García-Lario, P., Pottasch, S. R., et al. 1993c, A\&A, 267, L19

Parthasarathy, M., García-Lario, P., de Martino, D., et al. 1995, A\&A, 300, L25

Parthasarathy, M., Vijapurkar, J., \& Drilling, J. S. 2000a, A\&AS, 145, 269

Parthasarathy, M., García-Lario, P., Sivarani, T., et al. 2000b, A\&A, 357,241

Pègouriè, B. 1988, A\&A, 194, 335

Pottasch, S. R. 1992, A\&AR, 4, 215

Reed, B. C. 1998, ApJS, 115, 271

Ridgway, S. T., Carbon D. F., \& Hall, D. N. 1978, ApJ, 225, 138

Rieke, G. H., \& Lebofsky, M. J. 1985, ApJ, 288, 618

Ryans, R. S. I., Dufton, P. L., Mooney, C. J., et al. 2003, A\&A, 401, 1119

Schaeidt, S. G., Morris, P. W., Salama, A., et al. 1996, A\&A, 315, L55

Schild, R. E., Garrison, R. F., \& Hiltner, W. A. 1983, AJ, 88, 67

Schlegel, D. J., Finkbeiner, D. P., \& Davis, M. 1998, ApJ, 500, 525

Schmidt-Kaler, Th. 1982, in Landolt-Börnstein: Numerical Data and Functional Relationships in Science and Technology, vol. 2b, ed. K. Schaifers, \& H. H. Voigt (Berlin: Springer-Verlag)

Schönberner, D. 1983, ApJ, 272, 708
Schönberner, D., \& Blöcker, T. 1993, in Luminous High-Latitude Stars, ed. Sasselov, ASP Conf. Ser., 45, 337

Schwarz, H. E., Corradi, R. L. M., \& Melnick, J. 1992, A\&AS, 96, 2

Siebenmorgen, R., Zijlstra, A. A., \& Krügel, E. 1994, MNRAS, 271, 449

Swinyard, B. M., Clegg, P. E., Ade, P. A. R., et al. 1996, A\&A, 315, L43

Swinyard, B. M., Burgdorf, M. J., Clegg, P. E., et al. 1998, Proc. SPIE, 3354,888

Szczerba, R., Henning, Th., Volk, K., Kwok, S., \& Cox, P. 1999, A\&A, 345, L39

Tielens, A. G. G. M. 1983, ApJ, 271, 702

Treffers, R. R., \& Cohen, M. 1984, ApJ, 188, 545

Tylenda, R., \& Stasińska, G. 1994, A\&A, 288, 897

Volk, K., \& Kwok, S. 1989, ApJ, 342, 345

Volk, K., Xiong, G. Z., \& Kwok, S. 2000, ApJ, 530, 408

Volk, K., Kwok, S., \& Hrivnak, B. J. 2002, ApJ, 567, 412

Waters, L. B. F. M., Molster, F. J., de Jong, T., et al. 1996, A\&A, 315, L361

Waters, L. B. F. M., Beintema, D. A., Zijlstra, A. A., et al. 1998, A\&A, 331, L61

Waters, L. B. F. M., \& Molster, F. J. 1999, IAU Symp., 191, 209, ed. T. Le Bertre, A. Lébre, \& C. Waelkens

Whittet, D. C. B. 2003, Dust in the Galactic Environment, Second Edition (Institute of Physics Publishing), 226 